\title{
Presymptomatic cortical thinning in familial Alzheimer disease
}

\author{
A longitudinal MRI study \\ OPEN
}

Philip S.J. Weston, MRCP

Jennifer M. Nicholas, $\mathrm{PhD}$

Manja Lehmann, PhD Natalie S. Ryan, MRCP Yuying Liang, MRCP Kirsty Macpherson, BA Marc Modat, PhD Martin N. Rossor, FMedSci

Jonathan M. Schott, FRCP

Sebastien Ourselin, PhD Nick C. Fox, FMedSci

Correspondence to Dr. Weston:

philip.weston.11@ucl.ac.uk

Supplemental data at Neurology.org

\section{ABSTRACT}

Objective: To identify a cortical signature pattern of cortical thinning in familial Alzheimer disease (FAD) and assess its utility in detecting and tracking presymptomatic neurodegeneration.

Methods: We recruited 43 FAD mutation carriers-36 PSEN1, 7 APP (20 symptomatic, 23 presymptomatic)-and 42 healthy controls to a longitudinal clinical and MRI study. T1-weighted MRI scans were acquired at baseline in all participants; 55 individuals (33 mutation carriers; 22 controls) had multiple (mean 2.9) follow-up scans approximately annually. Cortical thickness was measured using FreeSurfer. A cortical thinning signature was identified from symptomatic FAD participants. We then examined cortical thickness changes in this signature region in presymptomatic carriers and assessed associations with cognitive performance.

Results: The cortical signature included 6 regions: entorhinal cortex, inferior parietal cortex, precuneus, superior parietal cortex, superior frontal cortex, and supramarginal gyrus. There were significant differences in mean cortical signature thickness between mutation carriers and controls 3 years before predicted symptom onset. The earliest significant difference in a single region, detectable 4 years preonset, was in the precuneus. Rate of change in cortical thickness became significantly different in the cortical signature at 5 years before predicted onset, and in the precuneus at 8 years preonset. Baseline mean signature thickness predicted rate of subsequent thinning and correlated with presymptomatic cognitive change.

Conclusions: The FAD cortical signature appears to be similar to that described for sporadic AD. All component regions showed significant presymptomatic thinning. A composite signature may provide more robust results than a single region and have utility as an outcome measure in presymptomatic trials. Neurology ${ }^{\circledR} 2016 ; 87: 2050-2057$

\section{GLOSSARY}

$\mathbf{A D}=$ Alzheimer disease; $\mathbf{C D R}=$ Clinical Dementia Rating; $\mathbf{E Y O}=$ estimated years to symptom onset; $\mathbf{F A D}=$ familial Alzheimer disease; MMSE = Mini-Mental State Examination; RMT $=$ Recognition Memory Test; VOSP $=$ Visual Object and Space Perception battery.

There is great interest in testing potential disease-modifying treatments for Alzheimer disease (AD) prior to the onset of symptoms. ${ }^{1,2}$ In order to facilitate this, robust and sensitive methods are needed to identify at-risk individuals, stage their disease, and track progression. ${ }^{3,4}$

Studies of volumetric MRI, using automated cortical thickness measurement, have suggested that sporadic $\mathrm{AD}$ is associated with cortical thinning in a relatively consistent pattern, or "signature," of cortical regions. ${ }^{5,6}$ A composite signature is likely to be more robust to interindividual variation than using a single region alone. We were interested in seeing if a similar cortical signature pattern of loss could be used to track changes presymptomatically in autosomal dominant familial $\mathrm{AD}(\mathrm{FAD})$.

FAD shares many features, both pathologically and clinically, with the more common sporadic form of the disease. ${ }^{7} \mathrm{FAD}$ genetic mutation carriers have relatively predictable ages at

\footnotetext{
From the Dementia Research Centre (P.S.J.W., J.M.N., M.L., N.S.R., Y.L., K.M., M.M., M.N.R., J.M.S., N.C.F.), UCL Institute of Neurology; Transitional Imaging Group (M.M., S.O.), Centre for Medical Image Computing, University College London; and London School of Hygiene and Tropical Medicine (J.M.N.), UK.

Go to Neurology.org for full disclosures. Funding information and disclosures deemed relevant by the authors, if any, are provided at the end of the article. The Article Processing Charge was paid by the MRC.

This is an open access article distributed under the terms of the Creative Commons Attribution License 4.0 (CC BY), which permits unrestricted use, distribution, and reproduction in any medium, provided the original work is properly cited.
} 
onset, ${ }^{8,9}$ which provides opportunity for prospective longitudinal study of individuals prior to the onset of clinical AD. Previous studies identified reductions in brain volumes before symptom onset, with longitudinal rates of change appearing more sensitive than crosssectional measurement. ${ }^{10,11}$

We report the results of a prospective longitudinal study of cortical thickness in FAD mutation carriers. We first describe the identification of a cortical signature of FAD,

Figure 1 The familial Alzheimer disease (FAD) cortical signature
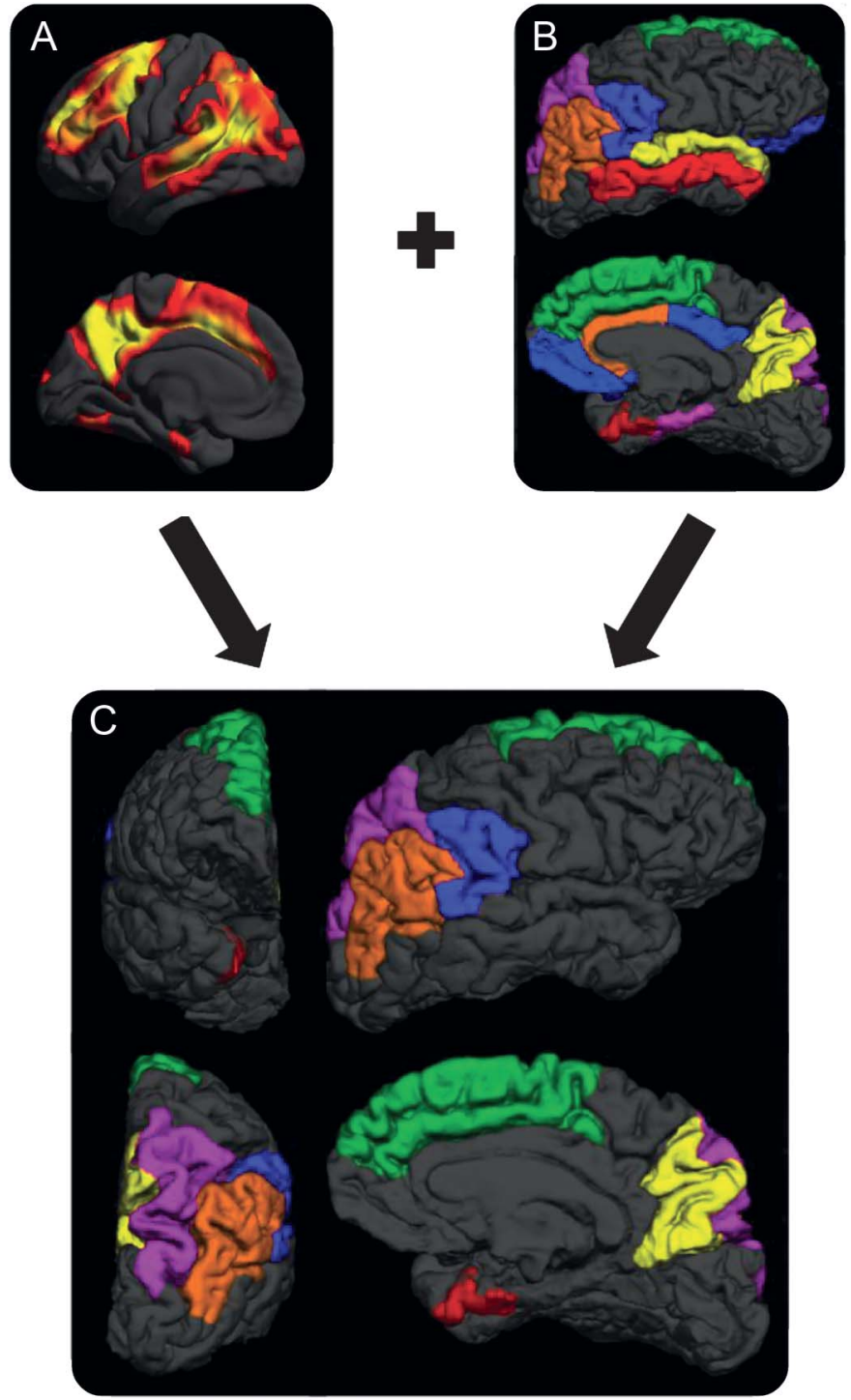

(A) Results of FreeSurfer analysis indicate regions exhibiting the most significant cortical thinning in the mildly symptomatic individuals with FAD from our cohort $(p<0.01$, familywise error corrected). (B) The results of a literature search show regions previously highlighted as showing significant atrophy in FAD. We identified regions that were positive in both the literature-driven and data-driven approaches, and included only these regions in our final FAD cortical signature (C). A total of 6 regions were included: entorhinal cortex (red), inferior parietal cortex (orange), precuneus (yellow), superior frontal cortex (green), superior parietal cortex (purple), and supramarginal gyrus (blue). determined in symptomatic participants, and then assess the utility of this approach in detecting presymptomatic change, in terms of both absolute thickness and rates of thinning. We then assess if baseline thickness can predict subsequent thinning and its correlation with preclinical cognitive changes.

METHODS Standard protocol approvals, registrations, and patient consents. The study was approved by the local research ethics committee and all participants provided written informed consent.

Participants. We recruited 85 participants between 2009 and 2014 to an ongoing longitudinal study of FAD at the Dementia Research Centre, University College London (appendix e-1 at Neurology.org). Forty-three participants were carriers of pathogenic mutations in either the presenilin 1 (PSEN1) $(\mathrm{n}=$ $36)$ or amyloid precursor protein $(A P P)(\mathrm{n}=7)$ genes and 42 were healthy controls (including 15 gene-negative siblings of the mutation carriers). The mutation carrier group included a total of 14 different PSEN1 mutations and 4 different APP mutations. At baseline, 20 of the mutation carriers were symptomatic and 23 were presymptomatic.

There were 58 participants ( 43 mutation carriers and the 15 noncarrier siblings) from families affected by FAD, with 28 different families represented. For these participants, genetic testing was performed to determine the presence or absence of a mutation. Genetic data were provided only to designated individuals performing the statistical analysis, thus ensuring that both the participants and the clinicians assessing them remained blind to their genetic status.

At each timepoint, approximately annually, participants underwent MRI, neurologic examination, detailed neuropsychological assessment, and assessment with the Mini-Mental State Examination (MMSE) and Clinical Dementia Rating scale (CDR). The neuropsychological test battery comprised measures of general intellectual functioning (Wechsler Abbreviated Scale of Intelligence); verbal and visual recognition memory (Recognition Memory Test [RMT] for words and faces); short-term memory (forward digit span); executive function (backwards digit span); naming (Graded Naming Test); calculation (Graded Difficulty Arithmetic Test); and visuoperceptual skills (object decision test from the Visual Object and Space Perception battery [VOSP]). All individuals identified a close informant who was interviewed separately to gain a collateral history. Individuals were defined as symptomatic if consistent symptoms of cognitive decline were reported by the participant or their informant and the CDR was $>0$. Estimated years to symptom onset (EYO) was calculated for the presymptomatic mutation carriers by subtracting the participant's current age from the age at which the affected parent first developed symptoms.

MRI acquisition. All participants at all timepoints were scanned on the same 3T S TIM Trio scanner using a 32channel phased-array head coil. A sagittal 3D magnetizationprepared rapid gradient echo T1-weighted volumetric MRI (echo time/repetition time/inversion time $=2.9 / 2,200 / 900 \mathrm{~ms}$, dimensions $256 \times 256 \times 208$, voxel size $1.1 \times 1.1 \times 1.1 \mathrm{~mm}$ ) was acquired.

Image analysis. We measured cortical thickness across the cortical mantle at approximately 300,000 vertices using FreeSurfer v5.30's (surfer.nmr.mgh.harvard.edu) cross-sectional pipeline. 
The procedure used by FreeSurfer for the surface construction has been described and validated previously. ${ }^{12,13}$ Two modifications to the standard FreeSurfer processing stream were undertaken: a locally generated brain mask was used for skull stripping and ventricular segmentations were added to the white matter mask to improve cortical segmentation. Cortical thickness was smoothed with a 20-mm full-width at half-maximum kernel. All cortical segmentations were visually inspected. Mean cortical thickness values were calculated for 34 parcellated cortical regions bilaterally.

Identifying the FAD cortical signature. We first undertook a detailed literature review in PubMed, using the search terms (familial Alzheimer disease) or (autosomal dominant Alzheimer disease) and (cortical thickness) or (cortical volume), to identify the regions previously found to undergo significant cortical atrophy in FAD (appendix e-1, table e-1). In addition, we separately analyzed data from our own cohort, using a conservative statistical threshold ( $p<0.001$, family-wise error corrected), to identify those regions that demonstrated the most significant bilateral thinning in the baseline scans of the 10 mildest (according to MMSE) symptomatic participants (appendix e-1). We then combined the results of these literature-driven and data-driven approaches, and only included regions in the final cortical signature that were identified by both methods (figure 1).

Longitudinal mixed-effects modeling. We applied a longitudinal linear mixed effects framework to model cortical thickness in the signature regions using data from all mutation carriers and noncarriers at all available timepoints (appendix e-1). The model included fixed effects for age, sex, mutation carrier status, and polynomial terms for EYO; a family level random effect for intercept; and participant level random effects for intercept, $\mathrm{EYO}$, and mutation carrier status. Of the 85 participants, 55 in dividuals ( 33 mutation carriers and 22 controls) had scans at multiple longitudinal timepoints (mean 2.9, maximum 6; mean \pm SD interscan interval $=448 \pm 172$ days). This longitudinal mixed effects approach was used to assess longitudinal change in both absolute cortical thickness and rates of change in cortical thickness.

In addition, we used linear mixed effects models to investigate whether lower baseline thickness was associated with increased rate of subsequent thinning, using data from the 33 mutation carriers who had scans at at least 2 timepoints. In this model, rate of subsequent thinning was estimated by a fixed effect for duration of follow-up. A fixed effect for interaction between baseline cortical thickness and duration of follow-up estimated the strength of association between the observed baseline value and rate of subsequent thinning. The model included a fixed effect for sex, family level random effect for intercept, and participant level random effects for intercept and duration since baseline (appendix e-1). ${ }^{14}$

Table 1 Demographics and baseline clinical characteristics of the sample

$\begin{array}{llll} & \text { Controls } & \text { Presymptomatic } & \text { Clinically affected } \\ \text { No. } & 42 & 23 & 20 \\ \text { Age, y (SD) } & 43.9(9.4) & 38.7(7.8) & 48.5(8.8) \\ \text { Sex, M/F } & 18 / 24 & 9 / 14 & 6 / 14 \\ \text { EYO, y (SD) } & - & 7.5(5.4) & - \\ \text { Global CDR (SD) } & 0 & 0 & 1.1(0.43)\end{array}$

Abbreviations: $\mathrm{CDR}=$ clinical dementia rating scale; $\mathrm{EYO}=$ estimated years to onset.
Assessing association between cortical signature change and neuropsychological performance. To assess the clinical implications of thinning within the cortical signature regions, we analyzed associations between cortical thickness and performance on neuropsychological testing in mutation carriers, both before and after symptom onset. Spearman correlation coefficients were used to assess cross-sectional correlation between mean cortical thickness across the 6 signature regions at baseline and score on each focal neuropsychological test. In addition, the following specific correlations were examined, based on hypothesized associations between specific regions and specific focal tests:

1. RMT (words and faces) and entorhinal cortex thickness.

2. Digit span forwards and superior frontal cortex, supramarginal gyrus, and inferior parietal cortex thickness.

3. Digit span backwards and superior frontal cortex thickness.

4. Arithmetic and supramarginal gyrus and inferior parietal cortex thickness.

5. VOSP object decision and supramarginal gyrus, inferior parietal cortex, and superior parietal cortex thickness.

As well as examining the above correlations among all mutation carriers, secondary analyses examined the correlation between cortical thickness and neuropsychological test scores in presymptomatic mutation carriers only. Cluster-adjusted (robust) $p$ values were used to account for clustering of individuals within families. All analyses used STATA v13.0 or later.

RESULTS The cortical signature. Mutation carriers and noncarriers were well-matched for age (43.2 years [SD 9.4] vs 43.9 [9.4]) and sex (15 male/28 female vs 18 male/24 female). The group demographics, with mutation carriers broken down into symptomatic and presymptomatic, are shown in table 1.

Six cortical regions were identified as making up the FAD cortical signature (figure 1): the entorhinal cortex, the inferior parietal cortex, the precuneus, the superior frontal cortex, the superior parietal cortex, and the supramarginal gyrus.

Presymptomatic cortical thinning detectable across the cortical signature. Our analysis of longitudinal cortical thickness change found that, for all 6 cortical signature regions, there was strong evidence of a difference in cortical thickness between mutation carriers and controls ( $p<0.0001$ for all regions) and for a difference in rates of change in cortical thickness $(p<$ 0.0001 for all regions). Combining all signature regions to give a mean signature measure showed cortical signature thickness to be significantly lower in mutation carriers compared to controls by 3 years before predicted onset (figure 2, table 2). The earliest significant difference in cortical thickness in any individual region was detectable 4 years preonset, in the precuneus.

Earlier differences between mutation carriers and controls were apparent, for all 6 signature regions, when comparing the rate of change in cortical thickness, rather than the absolute cortical thickness value alone (figure 2, table 2). Rates of 

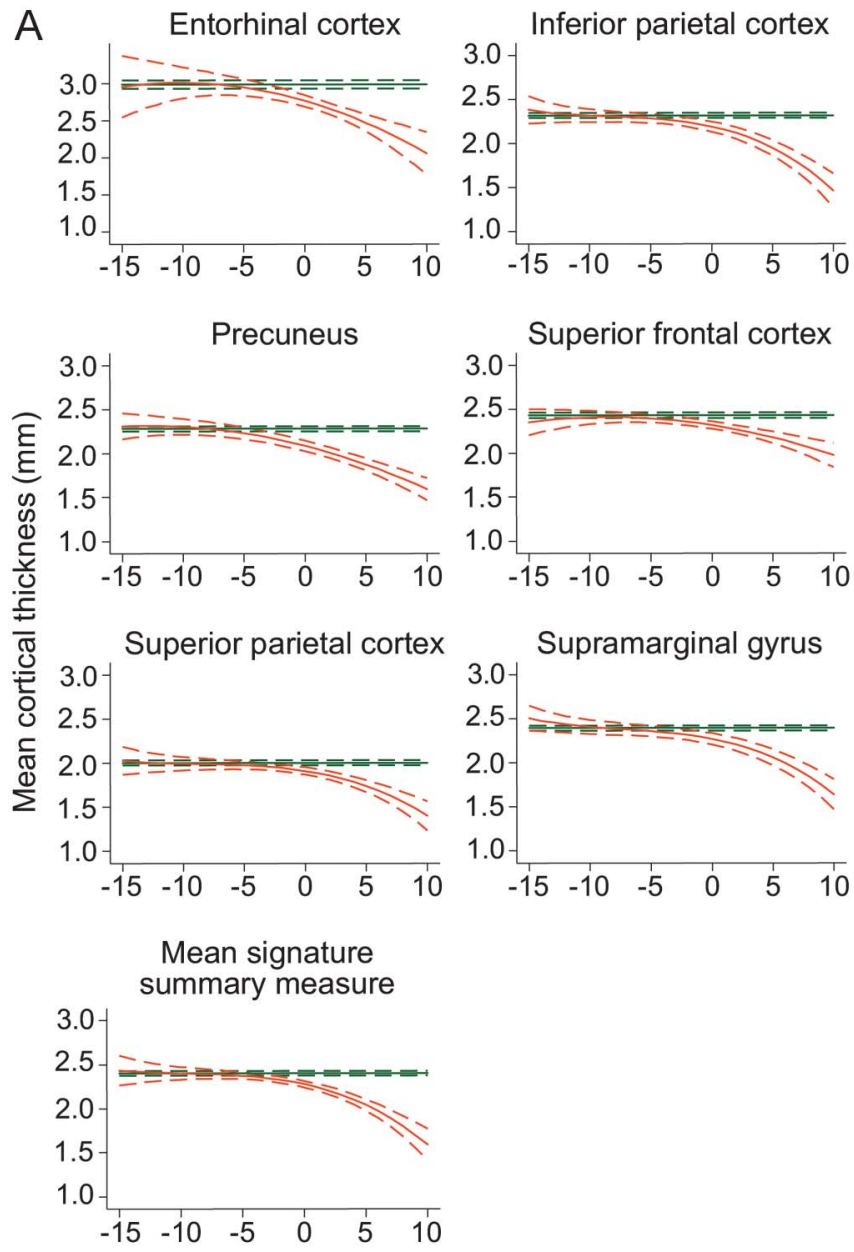

Estimated years to/from symptom onset
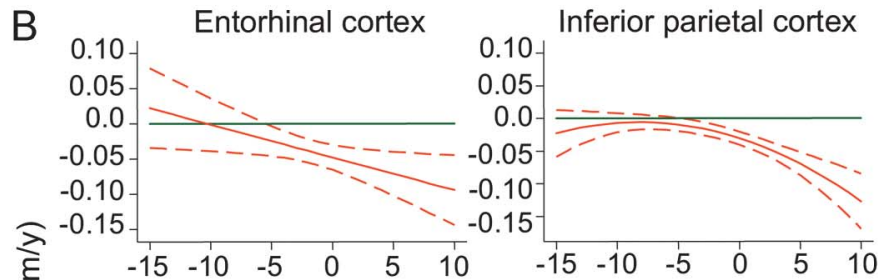

Precuneus

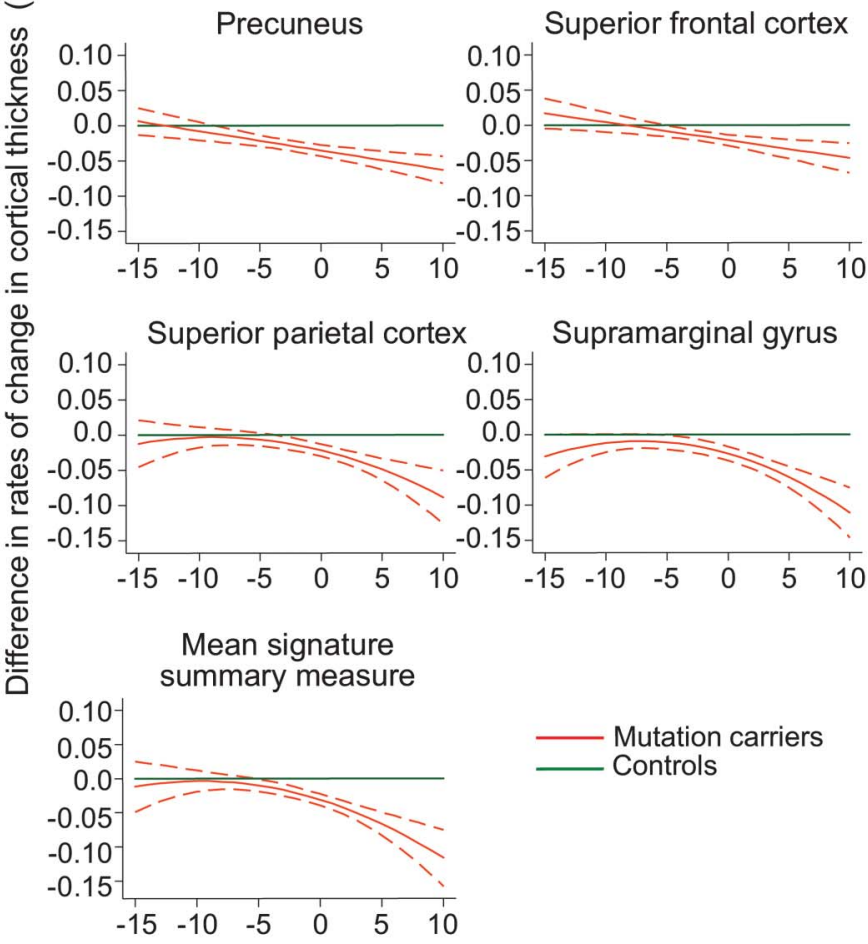

Estimated years to/from symptom onset

Graphs compare mutation carriers and controls for (A) absolute cortical thickness value and (B) difference in rate of change in cortical thickness, with the $x$-axes representing estimated time to/from onset of progressive cognitive symptoms. Graphs are shown for each cortical signature region as well as the mean signature summary measure. Dotted lines indicate $95 \%$ confidence intervals.

thinning in the mean signature summary measure become nominally higher in mutation carriers compared with controls at around 9 years before predicted onset, with the difference becoming statistically significant by 5 years before predicted onset. The earliest significant difference in rate of change in an individual region was again observed in the precuneus, with significant differences in rates of thinning seen at 8 years preonset. A paired $t$ test, including all 6 signature regions, found the change in rate to be significantly earlier than the change in absolute thickness $(p=0.005)$.

Across all mutation carriers, lower baseline cortical thickness predicted greater thinning in the subsequent follow-up period. This association was stronger for the mean signature summary measure than for any region individually (table 2). A significant association remained between mean signature thickness and future decline even when only presymptomatic carriers were assessed ( $p=0.022$ ); a similar association was found in this group for the precuneus $(p=0.029)$.

Correlation between cortical signature thickness and neuropsychological performance. Across all mutation carriers, there were significant positive correlations between the mean cortical signature summary measure and cognitive performance across tests of a wide range of cognitive modalities (table 3). For each of these tests, there was also significant correlation between test performance and cortical thickness in specific hypothesized individual cortical regions.

Looking at the presymptomatic participants only (median time to onset 8 years), there remained a statistically significant correlation between cortical thickness and performance in both verbal recognition memory and arithmetic, but not with other domains (table 3). 
Table 2 Longitudinal cortical thinning in the cortical signature regions

\begin{tabular}{|c|c|c|c|c|c|}
\hline & \multirow[b]{2}{*}{$\begin{array}{l}\text { Cortical thickness } \\
\text { (MC vs NC), earliest } \\
\text { significant }(p<0.05) \\
\text { difference }(E Y O)\end{array}$} & \multirow[b]{2}{*}{$\begin{array}{l}\text { Rate of change } \\
\text { (MC vs NC), earliest } \\
\text { significant }(p<0.05) \\
\text { difference }(E Y O)\end{array}$} & \multicolumn{3}{|c|}{$\begin{array}{l}\text { Association between baseline } \\
\text { cortical thickness and subsequent } \\
\text { rate of thinning (MC only) }\end{array}$} \\
\hline & & & $\begin{array}{l}\text { Coefficient ( } \mathrm{mm} / \mathrm{y} \\
\text { for every additional } \\
\mathrm{mm} \text { at baseline) }\end{array}$ & $95 \% \mathrm{Cl}$ & $p$ Value \\
\hline $\begin{array}{l}\text { Mean signature } \\
\text { summary measure }\end{array}$ & -3 & -5 & 0.184 & 0.063 to 0.305 & 0.0028 \\
\hline Entorhinal cortex & -2 & -5 & 0.115 & -0.104 to 0.335 & 0.30 \\
\hline Inferior parietal cortex & -3 & -4 & 0.139 & 0.036 to 0.242 & 0.0080 \\
\hline Precuneus & -4 & -8 & 0.105 & 0.029 to 0.180 & 0.0067 \\
\hline Superior frontal cortex & -3 & -5 & 0.162 & 0.038 to 0.286 & 0.011 \\
\hline Superior parietal cortex & -2 & -3 & 0.119 & 0.036 to 0.202 & 0.0049 \\
\hline Supramarginal cortex & -2 & -5 & 0.081 & 0.009 to 0.152 & 0.027 \\
\hline
\end{tabular}

Abbreviations: $\mathrm{Cl}=$ confidence interval; $\mathrm{EYO}=$ estimated years to symptom onset.

A comparison between mutation carriers $(\mathrm{MC})$ and noncarriers $(\mathrm{NC})$, indicating the number of years prior to predicted symptom onset when a significant difference is detectable in (1) cortical thickness and (2) the rate of change in cortical thickness. All regions show significant presymptomatic thinning, including a mean signature measure. For all regions, apart from the entorhinal cortex, there was a significant relationship between cortical thickness at a given timepoint and the subsequent rate of thinning, with lower baseline thickness associated with a greater rate of subsequent decline. This association was stronger for the mean cortical signature measure than for any individual region.

Despite the precuneus not being one of our prehypothesized regions for testing associations with cognitive decline, as it had demonstrated the earliest presymptomatic thinning of any individual region, we undertook a post hoc analysis to assess its association with presymptomatic cognitive performance. We found that, in presymptomatic individuals, precuneus thickness correlated with verbal recognition memory only (Spearman $R=0.48, p=0.018$ ).

DISCUSSION We report results of longitudinal cortical thickness change in presymptomatic FAD within a disease-specific cortical signature. The FAD cortical signature that we identified would appear to closely resemble that previously identified for sporadic $\mathrm{AD},{ }^{5}$ and included medial parietal, lateral parietal, and medial temporal regions. By then applying this cortical signature to an independent group of presymptomatic mutation carriers, we found that all 6 cortical signature regions showed significant thinning a number of years before the onset of cognitive symptoms.

Our findings suggest, consistent with previous studies of FAD mutation carriers, that the precuneus is one of the earliest regions to show significantly reduced cortical thickness, at approximately 4 years preonset. ${ }^{15,16}$ A previous cross-sectional study of $\mathrm{FAD}$, which applied the sporadic $\mathrm{AD}$ cortical signature (rather than a FAD-specific one), identified presymptomatic thinning in 3 of the regions identified in our study, including the precuneus, at an average of 6 years prior to onset. ${ }^{17}$ The reason for the slightly earlier precuneus thinning in this study may have been the increased homogeneity of the participants, with all mutation carriers having the same mutation (PSEN1 E280G).

Two other studies of presymptomatic FAD have reported an initial increase in cortical thickness, prior to later decline. ${ }^{18,19}$ The authors acknowledged that this was unexpected, and speculated that it may indicate a presymptomatic inflammatory process. Here, in a larger sample, we find no evidence to support an early cortical thickness rise. However, the possibility that cortical thickness may increase in the earliest presymptomatic phase-perhaps even earlier than the period our study spanned-before falling as individuals come closer to symptom onset cannot be excluded.

We found, across all 6 signature regions, that the rate of change in cortical thickness allowed significantly earlier detection of neurodegeneration than a cross-sectional measure of thickness at a single timepoint (table 2). Previous imaging studies have similarly suggested that rate of loss rather than absolute volume/thickness may be more sensitive to early pathologic change, ${ }^{10,11,16,20}$ although few FAD studies have measured longitudinal change in cortical thickness specifically. The timing of the increase in rate of decline in cortical thickness from our study was slightly earlier than found for other longitudinal volumetric imaging measures. ${ }^{11,21}$

We found that, in mutation carriers, thickness across the cortical signature regions correlated with cognitive performance (table 3). This was true even prior to the onset of symptomatic decline, in individuals who were on average 8 years from predicted 
Table 3 Correlations between baseline cortical thickness and cognitive performance

\begin{tabular}{|c|c|c|c|c|c|}
\hline \multirow[b]{2}{*}{ Test } & \multirow[b]{2}{*}{ Region } & \multicolumn{2}{|c|}{ All mutation carriers } & \multicolumn{2}{|c|}{$\begin{array}{l}\text { Presymptomatic carriers } \\
\text { only }\end{array}$} \\
\hline & & Spearman $R$ & $p$ Value & Spearman $R$ & $p$ Value \\
\hline \multirow[t]{2}{*}{ RMT faces } & Mean signature & 0.41 & 0.005 & 0.077 & 0.74 \\
\hline & Entorhinal & 0.40 & 0.009 & 0.020 & 0.93 \\
\hline \multirow[t]{2}{*}{ RMT words } & Mean signature & 0.67 & $<0.001$ & 0.65 & 0.001 \\
\hline & Entorhinal & 0.50 & 0.001 & 0.57 & 0.001 \\
\hline \multirow[t]{4}{*}{ Digit span forwards } & Mean signature & 0.45 & 0.014 & 0.13 & 0.66 \\
\hline & Superior frontal & 0.45 & 0.013 & 0.19 & 0.41 \\
\hline & Supramarginal & 0.49 & 0.004 & 0.23 & 0.47 \\
\hline & Inferior parietal & 0.47 & 0.009 & 0.082 & 0.78 \\
\hline \multirow[t]{2}{*}{ Digit span backwards } & Mean signature & 0.60 & $<0.001$ & 0.29 & 0.32 \\
\hline & Superior frontal & 0.57 & 0.001 & 0.18 & 0.45 \\
\hline \multirow[t]{3}{*}{ Arithmetic } & Mean signature & 0.77 & $<0.001$ & 0.43 & 0.075 \\
\hline & Supramarginal & 0.78 & $<0.001$ & 0.51 & 0.027 \\
\hline & Inferior parietal & 0.70 & $<0.001$ & 0.28 & 0.27 \\
\hline \multirow[t]{4}{*}{ VOSP } & Mean signature & 0.28 & 0.053 & 0.18 & 0.42 \\
\hline & Superior parietal & 0.15 & 0.37 & -0.028 & 0.90 \\
\hline & Inferior parietal & 0.23 & 0.17 & 0.089 & 0.70 \\
\hline & Supramarginal & 0.23 & 0.095 & 0.18 & 0.30 \\
\hline
\end{tabular}

Abbreviations: RMT = Recognition Memory Test; VOSP = Visual Object and Space Perception battery.

Spearman correlation coefficients are shown for performance on a number of focal cognitive tests and cortical thickness, in mutation carriers only. Correlations were assessed between each cognitive test and the mean signature summary measure, as well as for certain specific individual regions. To limit the number of comparisons, we constrained comparisons between focal tests and individual regions to only those for which we had a priori hypotheses (based on the previous literature). For each comparison, correlations were assessed both across the entire cohort and in presymptomatic individuals only. A total of 39/43 mutation carriers were included: 17/20 affected and 22/23 presymptomatic (i.e., 3 affected and 1 presymptomatic participant had missing neuropsychology data).

symptom onset. In the presymptomatic mutation carriers, the closest correlation was between cortical thickness and verbal episodic memory. This cognitive domain has previously been identified as being the earliest neuropsychological predictor of later decline. ${ }^{22-24}$ Compared to the precuneus - the cortical region that showed the earliest thinning - the statistical significance of the correlation between mean signature thickness and verbal episodic memory was greater by an order of magnitude.

Our findings also suggest that cortical signature thickness at a given timepoint may have prognostic value, in that it correlates significantly with future rate of decline. When analyzing all mutation carriers, assessing the mean thickness across a composite of preselected cortical regions provided stronger prediction of future decline than any single region alone (table 2 ), although when looking in presymptomatic individuals only the predictive value of the cortical signature and precuneus were similar. The finding that a multiregion signature provides predictive power and greater correlation with cognitive performance than any region in isolation is consistent with the concept that in AD there is early breakdown of a vulnerable but distributed neural network of different interconnected regions; the variability between individuals in the patterns of loss and sequence of recruitment of different regions with progression means that a composite signature region may be a more robust measure than a single region. ${ }^{25,26}$ A similar cortical signature approach that we use here for FAD has previously shown promise in identifying early symptomatic sporadic AD. 5,6,27

A composite cortical signature region may be useful in the recruitment to, and monitoring of, presymptomatic trials of disease-modifying therapies. ${ }^{2,3,7}$ The use of a marker of $\mathrm{AD}$ neurodegeneration, such as the cortical thickness signature, may allow presymptomatic disease staging by allowing prediction of how close an individual is to symptom onset. A cortical signature could also provide a means of quantifying loss over time, a potentially valuable outcome biomarker.

Compared to previous imaging studies of FAD, our study has a number of novel features that provide important insights into presymptomatic disease. We provide empiric evidence of the value of assessing 
presymptomatic longitudinal rate of change in cortical thickness, rather than just cross-sectional measurement; of the potential predictive value of using a composite signature region; and that cortical signature thickness is related to cognitive performance even prior to symptom onset.

This study has a number of limitations. In the selection of our cortical signature, we constrained the regions to select from those that had been identified previously in the literature as involved in FAD. While such an approach has the potential to introduce bias, combining a priori information from the literature with an independent dataset can produce more robust results, with greater face validity, when identifying specific features of interest. ${ }^{28}$ In presymptomatic mutation carriers, we used parental age at onset to estimate the age at which an individual will develop cognitive symptoms. While parental age at onset has been shown to correlate closely with actual age at onset and to closely relate to other methods of estimating disease onset (e.g., based on family mean or mutation mean age at onset), this remains a proxy measure, ${ }^{9}$ and it is only with longer term follow-up that the actual age at symptom onset can be confirmed.

A number of different genetic mutations were included in our cohort, including mutations of both the PSEN1 and APP genes. A degree of variability, in terms of both clinical phenotype and radiologic appearance, has been found between the different FAD mutations, both between genes and within the same gene, ${ }^{29,30}$ meaning that our cohort is likely to be heterogeneous. While this diversity means it may be more difficult to identify a common pattern, the inclusion of different mutations and genes means that the composite cortical signature identified is likely to be more robust to interindividual variability. Future validation of the utility of the cortical signature would be valuable, ideally in a large cohort such as the Dominantly Inherited Alzheimer's Network. ${ }^{31}$ Also of interest would be a direct comparison between thinning patterns in familial and sporadic cases.

FAD is characterized by cortical thinning that is detectable presymptomatically. Of the regions studied, the precuneus showed particularly early change. However, a composite signature of multiple vulnerable regions may provide more robust prediction of future decline, and closer correlation with preclinical cognitive change, than measuring a single region alone. Rates of thinning in the cortical signature became significantly abnormal about 2 years earlier than absolute cortical thickness. This imaging measure may have utility in presymptomatic trials.

\section{AUTHOR CONTRIBUTIONS}

P. Weston contributed to conceptualization and design of the study, collection, analysis, and interpretation of data, and drafting/revising the manuscript. J. Nicholas led the statistical analysis and interpretation of data and assisted in drafting/revising the manuscript. M. Lehmann contributed to study design, analysis and interpretation of data, and drafting/ revising the manuscript. N. Ryan contributed to collection of data and drafting/revising the manuscript. Y. Liang contributed to collection of data and drafting/revising the manuscript. K. Macpherson contributed to collection of data and drafting/revising the manuscript. M. Modat contributed to analysis and interpretation of the data. M. Rossor contributed to drafting/revising the manuscript. J. Schott contributed to design of the study and drafting/revising the manuscript. S. Ourselin contributed to design of the study and drafting/revising the manuscript. N. Fox contributed to conceptualization and design of the study, interpretation of results, and drafting/revising the manuscript.

\section{STUDY FUNDING}

Supported by the NIHR Queen Square Dementia BRU, The MRC Dementia Platform UK (MR/L023784/1 and MR/0070761), and the Medical Research Council (grant numbers MR/J014257/1, G0900421, G116/143). The Dementia Research Centre is supported by Alzheimer's Research UK, the Brain Research Trust, and The Wolfson Foundation. P.S.J.W. is funded by an MRC Clinical Research Training Fellowship (MR/M003108/1). N.S.R. is supported by a Brain Exit Fellowship. J.M.S. is supported by the NIHR UCL/H Biomedical Research Centre, EPSRC (EP/J020990/1), MRC (CSUB19166), and ARUK (ARUK Network 2012-6-ICE; ARUK-PG2014-1946). S.O. receives funding from the EPSRC (EP/M005332/1, EP/L016478/1, EP/J020990/1, EP/K005278), the MRC (MR/J01107X/1), the EU-FP7 project VPH-DARE@IT (FP7-ICT-2011-9-601055), the NIHR Biomedical Research Unit (Dementia) at UCL, and the National Institute for Health Research University College London Hospitals Biomedical Research Centre (NIHR BRC UCLH/UCL High Impact Initiative). N.C.F. and M.N.R. are NIHR Senior Investigators.

\section{DISCLOSURE}

P. Weston, J. Nicholas, M. Lehmann, N. Ryan, Y. Liang, K. Macpherson, M. Modat, and M. Rossor report no disclosures relevant to the manuscript. J. Schott has received research funding from Avid Radiopharmaceuticals (a wholly owned subsidiary of Eli Lilly), has consulted for Roche Pharmaceuticals and Eli Lilly, and serves on a Data Safety Monitoring Committee for Axon Neuroscience SE. S. Ourselin has received personal compensation for activities and research support from Ixico Ltd. He also received financial support for his research from Siemens Molecular Imaging, MIRADA Medical Solutions, and GE Healthcare. N. Fox reports that his research has received payment for consultancy or for image analysis services from Avid/Lilly, BMS, Elan/ Janssen, GE, Lundbeck, and Pfizer/Wyeth. Go to Neurology.org for full disclosures.

\section{Received January 14, 2016. Accepted in final form July 26, 2016.}

\section{REFERENCES}

1. Sperling RA, Jack CR Jr, Aisen PS. Testing the right target and right drug at the right stage. Sci Transl Med 2011;3:111-133.

2. Reiman EM, Langbaum JB, Fleisher AS, et al. Alzheimer's Prevention Initiative: a plan to accelerate the evaluation of presymptomatic treatments. J Alzheimers Dis 2011;26 (suppl 3):321-329.

3. Kozauer N, Katz R. Regulatory innovation and drug development for early-stage Alzheimer's disease. N Engl J Med 2013;368:1169-1171.

4. Sperling RA, Aisen PS, Beckett LA, et al. Toward defining the preclinical stages of Alzheimer's disease: recommendations from the National Institute on Aging-Alzheimer's Association workgroups on diagnostic guidelines for Alzheimer's disease. Alzheimers Dement 2011;7:280-292.

5. Dickerson BC, Bakkour A, Salat DH, et al. The cortical signature of Alzheimer's disease: regionally specific cortical thinning relates to symptom severity in very mild to mild 
$\mathrm{AD}$ dementia and is detectable in asymptomatic amyloidpositive individuals. Cereb Cortex 2009;19:497-510.

6. Bakkour A, Morris JC, Dickerson BC. The cortical signature of prodromal $\mathrm{AD}$ : regional thinning predicts mild $\mathrm{AD}$ dementia. Neurology 2009;72:1048-1055.

7. Bateman RJ, Aisen PS, De Strooper B, et al. Autosomaldominant Alzheimer's disease: a review and proposal for the prevention of Alzheimer's disease. Alzheimers Res Ther 2011;3:1.

8. Mullan M, Houlden H, Crawford F, Kennedy A, Rogues P, Rossor M. Age of onset in familial early onset Alzheimer's disease correlates with genetic aetiology. Am J Med Genet 1993;48:129-130.

9. Ryman DC, Acosta-Baena N, Aisen PS, et al. Symptom onset in autosomal dominant Alzheimer disease: a systematic review and meta-analysis. Neurology 2014;83: 253-260.

10. Fox NC, Warrington EK, Freeborough PA, et al. Presymptomatic hippocampal atrophy in Alzheimer's disease: a longitudinal MRI study. Brain 1996;119:2001-2007.

11. Ridha BH, Barnes J, Bartlett JW, et al. Tracking atrophy progression in familial Alzheimer's disease: a serial MRI study. Lancet Neurol 2006;5:828-834.

12. Dale AM, Fischl B, Sereno MI. Cortical surface-based analysis: I: segmentation and surface reconstruction. Neuroimage 1999;9:179-194.

13. Fischl B, Dale AM. Measuring the thickness of the human cerebral cortex from magnetic resonance images. Proc Nat Acad Sci USA 2000;97:11050-11055.

14. Byth K, Cox DR. On the relation between initial value and slope. Biostatistics 2005;6:395-403.

15. Benzinger TL, Blazey T, Jack CR, Jr, et al. Regional variability of imaging biomarkers in autosomal dominant Alzheimer's disease. Proc Natl Acad Sci USA 2013;110: E4502-E4509.

16. Knight WD, Kim LG, Douiri A, Frost C, Rossor MN, Fox NC. Acceleration of cortical thinning in familial Alzheimer's disease. Neurobiol Aging 2011;32:17651773.

17. Quiroz YT, Stern CE, Reiman EM, et al. Cortical atrophy in presymptomatic Alzheimer's disease presenilin 1 mutation carriers. J Neurol Neurosurg Psychiatry 2013;84: 556-561.

18. Fortea J, Sala-Llonch R, Bartres-Faz D, et al. Increased cortical thickness and caudate volume precede atrophy in PSEN1 mutation carriers. J Alzheimers Dis 2010;22 909-922.
19. Sala-Llonch R, Llado A, Fortea J, et al. Evolving brain structural changes in PSEN1 mutation carriers. Neurobiol Aging 2015;36:1261-1270.

20. Jack CR Jr, Shiung MM, Weigand SD, et al. Brain atrophy rates predict subsequent clinical conversion in normal elderly and amnestic MCI. Neurology 2005;65:1227-1231.

21. Yau WY, Tudorascu DL, McDade EM, et al. Longitudinal assessment of neuroimaging and clinical markers in autosomal dominant Alzheimer's disease: a prospective cohort study. Lancet Neurol 2015;14:804-813.

22. Fox NC, Warrington EK, Seiffer AL, Agnew SK, Rossor MN. Presymptomatic cognitive deficits in individuals at risk of familial Alzheimer's disease: a longitudinal prospective study. Brain 1998;121:1631-1639.

23. Linn RT, Wolf PA, Bachman DL, et al. The 'preclinical phase' of probable Alzheimer's disease: a 13-year prospective study of the Framingham cohort. Arch Neurol 1995; 52:485-490.

24. Lopera F, Ardilla A, Martinez A, et al. Clinical features of early-onset Alzheimer disease in a large kindred with an E280A presenilin-1 mutation. JAMA 1997;277:793-799.

25. Pievani M, de Haan W, Wu T, Seeley WW, Frisoni GB. Functional network disruption in the degenerative dementias. Lancet Neurol 2011;10:829-843.

26. Warren JD, Fletcher PD, Golden HL. The paradox of syndromic diversity in Alzheimer disease. Nat Rev Neurol 2012;8:451-464.

27. Dickerson BC, Wolk DA. Alzheimer's Disease Neuroimaging Initiative: biomarker-based prediction of progression in $\mathrm{MCI}$ : comparison of $\mathrm{AD}$ signature and hippocampal volume with spinal fluid amyloid-beta and tau. Front Aging Neurosci 2013;5:55.

28. Chu C, Hsu AL, Chou KH, Bandettini P, Lin C. Alzheimer's Disease Neuroimaging Initiative: does feature selection improve classification accuracy? Impact of sample size and feature selection on classification using anatomical magnetic resonance images. Neuroimage 2012;60:59-70.

29. Ryan NS, Rossor MN. Correlating familial Alzheimer's disease gene mutations with clinical phenotype. Biomark Med 2010;4:99-112.

30. Scahill RI, Ridgway GR, Bartlett JW, et al. Genetic influences on atrophy patterns in familial Alzheimer's disease: a comparison of APP and PSEN1 mutations. J Alzheimers Dis 2013;35:199-212.

31. Moulder KL, Snider BJ, Mills SL, et al. Dominantly Inherited Alzheimer Network: facilitating research and clinical trials. Alzheimers Res Ther 2013;5:48.

\section{Subspecialty Alerts by E-mail!}

Customize your online journal experience by signing up for e-mail alerts related to your subspecialty or area of interest. Access this free service by visiting Neurology.org/site/subscriptions/etoc.xhtml or click on the "E-mail Alerts" link on the home page. An extensive list of subspecialties, methods, and study design choices will be available for you to choose from—allowing you priority alerts to cutting-edge research in your field! 


\section{Neurology}

Presymptomatic cortical thinning in familial Alzheimer disease: A longitudinal MRI study

Philip S.J. Weston, Jennifer M. Nicholas, Manja Lehmann, et al.

Neurology 2016;87;2050-2057 Published Online before print October 12, 2016

DOI 10.1212/WNL.0000000000003322

This information is current as of October 12, 2016

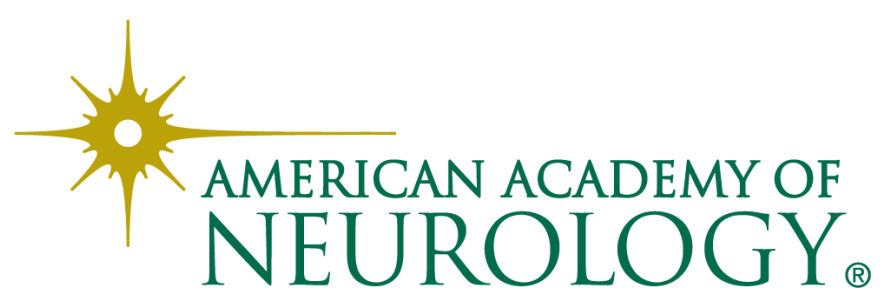




\section{Updated Information \& Services}

\section{Supplementary Material}

\section{References}

Citations

Subspecialty Collections

\section{Permissions \& Licensing}

Reprints including high resolution figures, can be found at: http://n.neurology.org/content/87/19/2050.full

Supplementary material can be found at: http://n.neurology.org/content/suppl/2016/10/12/WNL.0000000000003 322.DC1

This article cites 31 articles, 6 of which you can access for free at: http://n.neurology.org/content/87/19/2050.full\#ref-list-1

This article has been cited by 1 HighWire-hosted articles: http://n.neurology.org/content/87/19/2050.full\#\#otherarticles

This article, along with others on similar topics, appears in the following collection(s):

All Neuropsychology/Behavior

http://n.neurology.org/cgi/collection/all_neuropsychology_behavior Alzheimer's disease

http://n.neurology.org/cgi/collection/alzheimers_disease

Clinical trials Observational study (Cohort, $\overline{\text { Case control) }}$

http://n.neurology.org/cgi/collection/clinical_trials_observational_study cohort_case_control

Prognosis

http://n.neurology.org/cgi/collection/prognosis

Volumetric MRI

http://n.neurology.org/cgi/collection/volumetric_mri

Information about reproducing this article in parts (figures,tables) or in its entirety can be found online at:

http://www.neurology.org/about/about_the_journal\#permissions

Information about ordering reprints can be found online:

http://n.neurology.org/subscribers/advertise

Neurology ${ }^{\circledR}$ is the official journal of the American Academy of Neurology. Published continuously since 1951, it is now a weekly with 48 issues per year. Copyright (O 2016 American Academy of Neurology. All rights reserved. Print ISSN: 0028-3878. Online ISSN: 1526-632X.

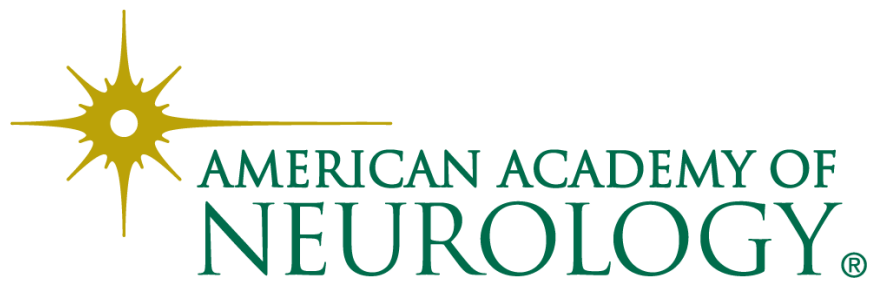

